\title{
Reorganization in Massive Multiagent Systems
}

\author{
Henry Hexmoor \\ Department of Computer Science, \\ Southern Illinois University, \\ Carbondale, IL 62901, USA \\ Email: \{hexmoor\}@cs.siu.edu
}

\section{BACKROUND}

Abstract - We have explored principled mechanisms for converting a hierarchical organization to an edge type organization. Other than structural differences, organizations differ in information flow network and information sharing strategies. Beyond current effort, many other types of organizational adaptation are possible and require much further research that we anticipate to remain for future work. This article lays the foundation for automatic organizational adaptation.

\section{INTRODUCTION}

Hierarchical command and control (C2) structures are ineffective (Alberts and Hayes, 2003). Power to the Edge $(\mathrm{PE})$ is an information and organization management philosophy that has superseded traditional organizational paradigms for C2. PE provides empowerment for edge members, as well as superior, interoperablem agile, shared awareness among all the members in the organization. PE provides adaptability in dynamic situations [6]. Man on the Loop (MOTL) complements PE with a set of computational tools for systematically altering organizational components for agile response to dynamics in large population multiagent systems [21, 25]. Reorganization is necessary to allow communities of agents or robots to reconfigure their organizational structure in agile response to changes in the environment.

We have explored principled mechanisms for converting a hierarchical organization to an edge type organization. Other than structural differences, organizations differ in information flow network and information sharing strategies. Many types of organizational adaptation are possible and require in-depth research that we anticipate to remain for our future work. This article lays the foundation for automatic organizational adaptation. We begin by outlining related work and background in section 2 . In section 3 we present an approach to reorganization. Section 4 describes an implementation of a simulated testbed that will help us validate our salient concepts. Preliminary results and conclusions are in sections 5 and 6 respectively.

\footnotetext{
$\square$ This work was supported by AFRL under contract FA8750-06-C-
}

In highly dynamic operational environments, it is desirable for multi-agent systems (MAS) to be capable of self-directed structural reorganization. The organizational structure of MAS, whether based on a graph, hierarchy, federation, or other form, dictates the communication interactions between agents as well as the distribution of roles and authority throughout the system. When motivated to adapt, agents should do so without human intervention and in a manner that improves the overall performance of the system. Several motivating factors for reorganization will be discussed here as will current proposed methods for performing dynamic reorganization in MAS. Current research in the area of dynamic reorganization in multi-agent systems has yielded a few approaches in dealing with the problem of adaptation in uncertain and often hostile environments. Given the intelligent and autonomous nature of agents in MAS, individual agents must be capable of locally adapting their interconnection schemes with respect to other agents in the MAS. In addition, agents must be able to accept new roles and to comply with any restrictions or laws associated with these roles. Limitations of communication bandwidth, imposed by the environment or power considerations, or explicitly mandated due to security concerns, implies that agents may not, and probably will not, have a complete picture of the effectiveness and efficiency of the global system. With these limitations in mind, agents must adapt based on local perception of global performance [17].

The impetus directing reorganization varies widely. Common adaptation triggers are based on estimates of the overall performance of the MAS, timelines specifying that reorganization should take place at scheduled intervals, or structural requirements. Matson and DeLoach have described other adaptation triggers related to roles and contrast adaptation for timeline-based efficiency and for quality-based effectiveness [24]. They illustrate three scenarios resulting in a need for reorganization. The first of these relates to the situation in which the organizational objective demands a role that has not yet been assigned. In this situation, there may or may not be extra agents available to accept the required role. Second, a role that is currently 
assumed by an agent may be relinquished by that agent, resulting in incomplete role distribution as with the first scenario. Third, an agent may be forced to relinquish a role due to some internal fault or as a result of malicious activity. In this case, the system may not be informed of the need to reassign the lost role. similar triggers are described in [14]. These include allocation, reallocation, and exchange. In an allocation scenario, an agent has completed its task and is allocated a new task. In the reallocation scenario, an agent prematurely terminates its current task and is allocated a new one. In the final scenario, exchange, two agents swap tasks. Regardless of the adaptation trigger driving reorganization of a MAS, the desired outcome remains efficient completion of the system objective. A system of reorganization based on dynamic capability evaluation is presented in [26].

Matson and DeLoach's approach to reorganization of MAS first involves the evaluation of the system's ability to perform a desired task. Based on this evaluation, agents may decide to either proceed to satisfy the organizational goals, relax some goals, or abandon the process of reorganization and task acceptance altogether. The foundation of this approach is an organizational model consisting of goals, roles, agents, and capabilities. Based on this model, certain evaluative constraints are applied to the process. First, there must exist knowledge of which agents are available for inclusion in the system. Second, it must be determined what necessary capabilities exist in order to satisfy the demands of a role. Third, an assessment of the capabilities of all available agents must be made to determine their respective qualifications for acceptance of a given role. To perform this step, the authors have devised a capability taxonomy rooted at the abstract level. Leaf nodes of this taxonomy represent concrete functions and capabilities of an agent, such as the types of sensors (sonar, infrared, etc.) and motivators (wheels, tracks, etc.) the agent is equipped with. Finally, limitations applied to roles must be taken into consideration. Considering these constraints, Matson and DeLoach formulated a six step evaluation process, which begins with the broad definition of system goals. Following this, the broad goals must be reduced into a simpler, structured format. Using this structured form of the system goals, the process determines all roles which will be required to complete the prescribed objectives. A general purpose method has been developed and discussed in [31]. This method is based on the organizational model for adaptive computational systems (OMACS) platform and has been shown to result in optimal network configurations. Another method for performing dynamic organization and reorganization is based on the principle of referral networks. [30] that describe such networks. In referral networks, agents make and sever connections with other agents in the system through the analysis of referrals provided by neighboring agents. An agent wishing to enter the network or alter its set of interconnections once within the network accepts referrals from surrounding agents. From these referrals, agents can form opinions regarding the quality of service provided by other agents and their respective trustworthiness. These are referred to as an agent's expertise and sociability [30].

Agents in referral networks use their knowledge of the trustworthiness and sociability of other agents in the system to decide which agents with which to sever communications or with which to add communication links. Agents that possess high trustworthiness and sociability rankings attract more agents. As the highly trusted and sociable agent gains connections with other agents, its degree increases and it therefore has a greater chance of being referred to other agents in the system. This, in turn, leads to a clustering of agents around the ones seen as the most fit. Fitness directly relates to trustworthiness and sociability. Clustering of this kind is linked closely to the concept of preferential attachment [5] and has been identified in many real-world networks, especially in the Internet. Referral networks are classical and useful, but other methods of reorganization exist. Some of these methods attempt to model biological and chemical organization methods. One such method, related to the concept of stigmergy, is referred to as the digital hormone model [27]. This model is based on the understanding that hormonal signals are used often in nature to form organizations of high complexity. In the digital hormone model (as it relates to agents or robots), agents emit activator or inhibitor signals, i.e. hormones, into their surroundings. Once diffused into neighboring agent regions, the agents in these regions combine the incoming hormone strengths with those already present in their area and adapt their behavior based on these recalculated hormone strengths. The actual reorganization process in the DHM requires four steps, which are repeated continuously, assumedly until some goal has been reached. These steps begin with agents assuming roles based on their abilities and associated rules which govern their behavior. Next, execution of roles takes place. This is followed by each agent transmitting and receiving digital hormones to and from their surrounding areas. The final step in this process involves updating each agent's view of the concentration of hormones in its surrounding area.

Some considerations to keep in mind when selecting an adaptation mechanism or creating a new one are the learning rate, stability, and global structure of the MAS formed by the mechanism [17]. Other considerations are given in [18]. In this, the authors present the question of which agents should be allowed to adapt in the event of a failure. Three possibilities are considered and include random agent sets, a single agent in the event of a team failure, and all neighboring agents in the event of a single node failure. Furthermore, the authors propose a candidate pool of available agents with which an adapting agent may establish a connection. These are limited to the set of all agents, exteammates of the adapting agent, or referred agents, as are used in referral networks. This is continues in [18] with this work by outlining a process by which agents adapt given the above noted constraints. The process begins with the construction of the candidate pool and proceeds with several filtering stages. Structural filtering and skill filtering are 
performed first, followed by degree filtering in which only candidates with the single highest degree are left to connect with the adapting agent.

A general overview of dynamic reorganization concepts and examines two metrics useful in examining MAS performance; society utility and agent utility is found in [16]. Society utility is further decomposed into the success of interactions, roles, and structures in the system. Agent utility is not clearly defined, as it differs from agent to agent in heterogeneous agent systems. In addition to these utility metrics, [15] classifies several types of reorganization "maneuvers." The first of these, pre-emptive reorganization, is a viable option in unpredictable environments where possible, or likely, events can be prepared for in order to take full advantage of them. Protective reorganization attempts not to take advantage of possible future events, but instead works to limit the negative effects of such events on the system. Exploitive reorganization takes place after the fact, and seeks to benefit from events that have already taken place. Finally, corrective reorganization attempts to lessen the damage caused by events which have previously occurred in order to maintain system usefulness. Specific methods for performing adaptation are not present in [16], but it provides many useful ideas for developing new methods or for elaborating on existing methods.

\section{An Organizational Model}

Next, we define salient attributes for a computational organization. We will begin by defining a set of parameters that characterize an organization. First, we will define capabilities.

1: A capability is basic agent ability with a degree in the range from 0.0 to 1.0 . We will denote degree of a capability c with $\mathrm{D}(\mathrm{c})$.

We assume that there is no decay in capability and agents can only increase their capability. Furthermore, we assume capabilities are mutually exclusive. Let $\mathrm{C}$ denote a set of capabilities, which are required in the system for performing all tasks. I.e., $\mathrm{C}=\left\{\mathrm{C}_{1}, \mathrm{C}_{2}, \ldots, \mathrm{C}_{\mathrm{n}}\right\} . \mathrm{C}$ is the set of all capabilities known by all agents. Each agent will possess each capability $\mathrm{c}_{\mathrm{i}}$ to a different degree and may improve it by learning. This provides us with an $\mathrm{n}$ dimensional space of capabilities. Let's call this a C-space [21]. Next, we will define roles.

2: An action role, denoted with $\mathrm{r}$, is a point in C-space that specifies a minimal capability profile to qualify an agent for the role.

For example, with two capabilities c1 and c2, $<0.1,0.5>$ is a role that an agent may adopt if it's capabilities c1 and c2 exceed 0.1 and 0,5 respectively [10].

To execute an action role, an agent senses its environment, picks the best rule to determine an action, and performs the action. Success or failure of actions performed are determined in the environment and not known a-priori. At best, an agent may determine a probability of success based on its role fitness to perform it. We'll call the rate of an agent's success its productivity.

Productivity is one of three components for an agent to determine its utility with respect to a goal. The second component is its synergy with others in that role. The third component is the level of fitness of an agent to the role. Fitness of an agent is the sum of ratios of its capability over required capabilities. We are now ready to define an agent's utility with respect to a role.

$$
\text { Productivity }=\text { preference }(A) * \text { fitness }(A, R)(1)
$$

3: The utility of an agent $A$, performing in a role $\mathrm{R}_{\mathrm{i}}$, denoted by $u\left(A, R_{i}\right)$, is a linear combination of its productivity, its synergy levels for that role, and its fitness, i.e.,

$$
\mathrm{u}(\mathrm{A}, \mathrm{R})=\mathrm{P}\left(\mathrm{A}, \mathrm{R}_{\mathrm{i}}\right)+\left[\left(1 / \operatorname{sizeof}\left(\mathrm{R}_{\mathrm{i}}\right)\right) * \sum \mathrm{s}(\mathrm{i}, \mathrm{j})\right]
$$

Utility as defined here is intended to denote the relative satisfaction of an agent with a role. If this value is sufficiently high, an agent will be content with its current role. However, if this utility value is low, the agent will be inclined to change its role. As we will discuss norms later in this report, using individual utility as a basis for role change assumes a selfish norm. In general, norms are prescribed by a user, which in turn will affect agent decision making. So far, we've considered role changes motivated by utilities alone. However, a major intuitive motivator for role exchange is opportunity. In general, opportunity is determined by analyzing environmental attributes that suggest the degree to which adoption of a role by an agent will contribute to system or individual productivities. As an example, a midfielder may see the ball near the opponent's goal and determine that it has a good chance of scoring if it played a forward. Real world computations like this are very rapid and continuous, [19]. Opportunistic computing has also entered technical domains [28].

Agents need to continually, mentally quantify potential margins of system or individual productivity gains against all possible roles they could adopt. Whichever role candidate will yield the highest marginal gain will be the next role the agent will wish to take. The agent's choice of next role is a proposal that need to be presented to the organization and once processed; the agent may proceed with adoption of it.

For a vacant role, two temporal constraints of the role augment the notion of opportunity. The first is the immediacy of the need to occupy the role. Each open role will specify the urgency of the role. For instance, the team captain will assign a temporal urgency for each vacant role to be filled. Agents who vie for a vacant position, must meet the urgency constraint. Agents must dynamically compute their capability to transition into an open role. Naturally, this 
capability differs from an agent's innate abilities to perform action. It is not a personality trait. Also, it is not a universal agility or flexibility to take on roles in general. This capability differs with respect to each role and depends on the environmental circumstances. The second temporal constraint is the duration after which the role becomes obsolete and there will not be a need to occupy it. If the validity time window ends, we say such a role is expired. Agents must account for this constraint and should only consider a role if it is not yet expired.

In contrast to an action role, a role that is decision oriented is a manager role. An example of a manager role is captain of a football team.

4: Rank of a role $\mathrm{r}_{\mathrm{i}}$ assigns a number to the role that reflects its relative importance in an institution. This is denoted by $\operatorname{Rank}\left(\mathrm{r}_{\mathrm{i}}\right)$.

This is a highly simplified model of a role's valuation in an institution. Using this we introduce a notion of role order. The function "Rank" may return any natural number. The smaller the number the more preferred the role. Role $r_{j}$ is the most preferred rank if $\operatorname{Rank}\left(r_{j}\right)=1$. Importance of a role is inversely proportional to its rank.

5: Role Ordering ( $\mathrm{RO}$ ) is an ordering of action roles. Each role is assigned a unique rank. I.e., $<\operatorname{Rank}\left(\mathrm{r}_{1}\right)$, Rank $\left(\mathrm{r}_{2}\right)$, .... , Rank $\left(r_{n}\right)>$ specifies role ordering where $\operatorname{Rank}\left(r_{i}\right)$ is the $\mathrm{i}^{\text {th }}$ position is the rank for $\mathrm{i}^{\text {th }}$ role.

If $\operatorname{Rank}\left(r_{i}\right)<\operatorname{Rank}\left(r_{j}\right)$ then role corresponding $r_{i}$ is preferred over the role $r_{j}$ that has a smaller rank. RO sets up a trajectory in C-space.

6: A norm is a convention in the form of a rule shared by all individuals. It may govern role adoption with a set of rules. We denoted the set of norms by $\mathrm{N}$.

For simplicity, we consider organizational norms to be mutually exclusive and non-overlapping. In this paper we will limit norms to rules that govern role change. An example of a norm that governs roles is that individuals incrementally improve their capabilities over time and are allowed to apply for a higher rank in the organization-- this is the promotion norm. Another norm that will govern roles is based on utilities. The selfish norm will only consider individual utilities whereas beneficent will only account for the society's benefits. We assume that norms are determined outside organizations. In our framework, Moe issues a norm.

7: A department, denoted by $\mathrm{D}_{\mathrm{i}}$, is a fixed number of agents who are performing the same role $\mathrm{R}_{\mathrm{i}}$. Each department will require a minimum number of individuals needed to occupy roles in that department at a given time. We assume that departments are static and will not change over time. If there is a significant change for a department such as the number of individuals required, a new organization is formed.
Since departments and roles have a one to one correspondence, ranking one prescribes an ordering on the other. We are now ready to define an organization.

8: An Organization is modeled as $\langle\mathrm{C}, \mathrm{R}, \mathrm{D}, \mathrm{N}\rangle$, which is capabilities, roles, departments, and norms.

When an organization is initially populated by individuals, each agent adopts a role depending on its capability level. Each agent will aspire to occupy a role that is highest ranked in the organization.

After an organization is populated, it will experience reorganization where self-motivated individuals may change roles based on the promotion norm. This is shown in Figure 1.

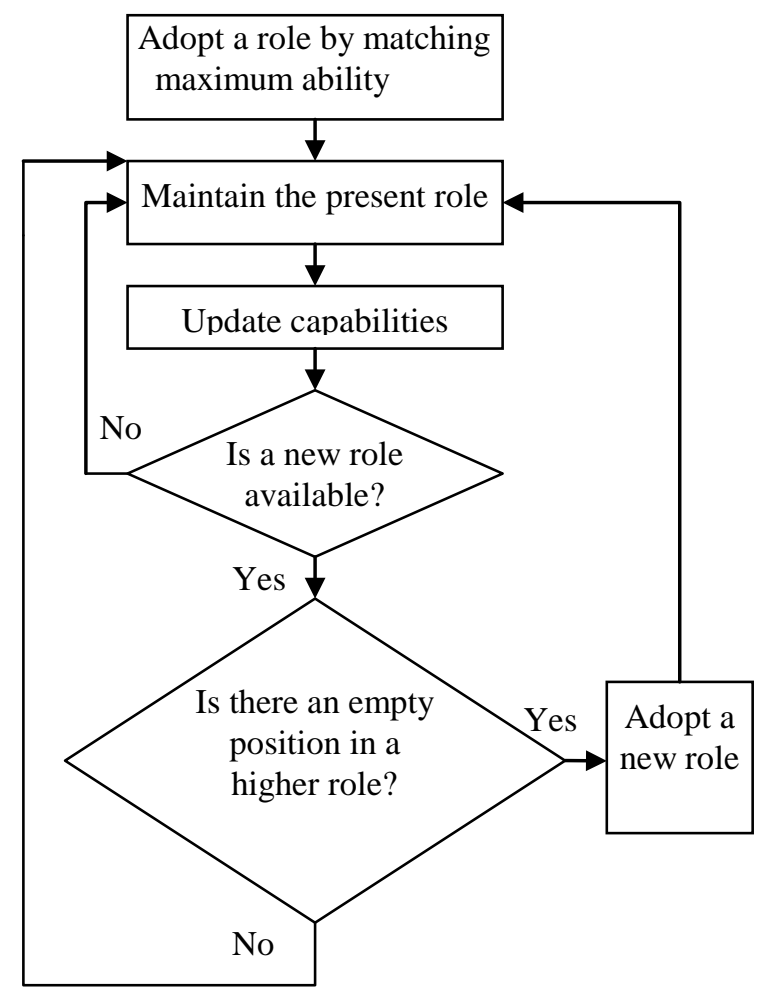

Figure 1. Flow chart for self-initiated promotion norm

We assume that individuals dynamically change roles and move to different departments. To capture a snaphot of organizational configuration we define a state that will be defined later.

9: At any given time, the configuration of individuals in departments of an organization is the state of that department.

States change as often as individuals change their roles. Therefore, there can be many state transitions over time. 
State transitions are most significantly affected by a norm change.

Thus far, our model of an organization lacks characteristic concepts of agents that will occupy it. In the next section we introduce these notions.

10: A synergy network among a group of agents is a graph among agents where the arcs represent a real value between 1.0 and 1.0 indicating negative or positive influence between pairs of agents. We will use $s(i, j)$ as an directed function that returns the synergy value between agents $i$ and $j$. Synergy is not reflexive and not symmetric.

Synergy between an agent agent A and another agent B is a degree of influence from B towards A. This can be positive or negative. We assume authority as a form of synergy. For example a manager role like a captain will have synergy towards players. Synergies change over time. One form of change is experience. Individuals in a department who interact with one another generally develop positive synergies towards one another that is proportional to the duration of time they remain the same department. However, this is not universally true and synergy change goes beyond departmental boundaries. What is important is how one agent's action enables another to perform its action.

Simply modeled, frequency of interactions between agents A and $\mathrm{B}$ that leads $\mathrm{B}$ to execute its role, increases synergy from $A$ to $B$. If $B$ is prohibited from executing its action due to A's action, then synergy decreases from A to B.

These changes in synergy cannot be simply determined from the organizational configuration alone. To recapitulate, Synergy changes are dynamic and dependent on interactions.

Beyond individual productiveness, an organization may produce something that is an emergent property and it is not attributed to a single individual. An example is scoring points that is a result of team work. Let's call this system productivity. Similar to an individual, we model a system utility that is a measure of the relative satisfaction of the organization.

11: Organizational utility is the sum of individual productivities plus balance of synergies among individuals in the organization.

12: A state of an organization is the combination of current, active norm and profile of roles occupying departments of the organization, i.e., composition of roles.

Since there are many possible role compositions and there are several possible norms, an organization can be in numerous states. A change in organizational role composition or a change in active norm will yield a state change. States of the system are completely observable and changes in states are under our control. Since state changes are not dependent on the sequences of past states, we can make the Markovian assumption. The nature of organizational state space fits a Markov decision process MDP. There are well known methods for solving MDPs such as the value iteration method [2].

\section{Implementation}

In order to illustrate the implementation of reorganization, we have designed a model that will be implemented using Netlogo. Netlogo is a java based cross-platform multi-agent programmable modeling environment for simulating natural and social phenomenon. It is freely available online at http://ccl.northwestern.edu/netlogo/.

The Domain which we will use is a simplified version of the game of Soccer

(i.e., see "http://en.wikipedia.org/wiki/Football_(soccer)”.

The game in our simulation is designed in the following fashion.

Capabilities: In this simulation we assume each player has a set of capabilities associated with him, which may or may not be unique. The capabilities are variables that are subject to change with the progress of the game. In our game each player from the start of the game maintains his capabilities at the same level. The set of capabilities each player has are threefold: Speed, Accuracy with handling the ball, Ability to kick the ball (far/near).

Speed: This represents the speed with which a player moves across a field. His ability to chase the ball and move the ball along.

Accuracy: This sets the players accuracy in handling the ball in the field. His ability to tackle with the ball when the ball is within his reach is decided by this parameter. Accuracy of a player ranges between $20^{\circ}-100^{\circ}$.

Kick: This ability of kicking a ball determines the distance a ball moves when a player kicks it.

The game is divided into a set of roles where each player can play only one role at a time. These are forward, Defender, Mid-fielder, and goal keeper. We omit details for brevity and space constraints.

Productivity: The productivity of each player is calculated according to the formula mention in the approach section the report although it had been adopted to each department to meet the requirement of the requirement of the department. The basis of calculation of productivity is mentioned in the above paragraphs describing the features of the department. The productivity of individual player is calculated and the productivity of the department is calculated basing on that. The team productivity is calculated considering the department productivities and the performance of the team as 
a whole. The productivities of the team are monitored in the graph in the user interface of the simulation.

Utility: the utility of each individual player performing in the designated role is calculated according to the earlier mentioned formula. The individual utility is how effective each person is performing in the designated role and the task at hand. We considered a randomly generated synergy while calculating the individual utilities. The department utility is calculated considering how effective those assigned individuals are at performing tasks and promoting the interests of the department. The utility of the team as whole has been calculated by considering the effectiveness of the departments performance in promoting the interests of the team , the synergies generated between the departments of the team and the overall performance of the team.

Norm: We implemented norm in this simulation. The norm in this game acts as the motivation or attitude with which the players play the game

We designed three norms, each of it differs from the others in unique way.

The norms implemented are Attack, Defend, Self-Gain. In the game the end user or MOTL gives the norm which governs the game.

Norms and reorganization:

Attack Norm: In this norm the players are expected to play with attack mode on their mind. The game is modifies such a way that there are more number of players in the forward department, four instead of three. With more number of players in the forward the game tends to be more aggressive at the cost of weakening the mid-field and defense departments. This is more offensive approach. The reorganization among the players is mainly motivated by the attack norm. The game starts normally and after a period of 500 clock cycles the system checks for any other player who has more capabilities to play in the forward. If it does not implicitly find one it calls for a reorganization and reassigns the roles to the players. The system does not look for best or worst performance it just does the reorganization until Moe is satisfied with it. Only Moe can instruct a system to stop reorganization. So Moe is the one who controls the extent to which she can permit reorganization.

Defend Norm: In this norm the players are expected to play in a defensive mode. The defense department has four players instead of regular three. With more players in the defense the game tends to be more defensive at the cost of loosing aggression; i.e., by weakening the mid-field we also reduce the support to the forward department. After a period of 500 clock cycles, the system checks for players who can be more effective to play in the defense department, and calls for a reorganization. The system this way keeps reorganizing until it receives any instruction from Moe.

Selfish Norm: With this norm player's play in the regular department layout configurations. Instead of caring for the team, they play to maximize their productivity and improve their utility. When reorganization is initiated, the system checks if a player is suitable for the role she is playing. If the system considers the player can perform better in another role it calls for reorganization. This is performed by computing the average productivity of the department. If the player falls below the department average she is underperforming so she is replaced. The system maintains reorganizing until Moe aborts it.

Synergy: In this simulation, we take the average of the department's average productivity and compare it with the individual synergy. If player's productivity is more than the departments' productivity we assume she has positive synergy. If she is performing below his capability we assume that she has negative synergy. Each player in the game has a synergy with respective to their department.

\section{Results}

We consider two cases for exhibiting results of using our simulation

Case 1:

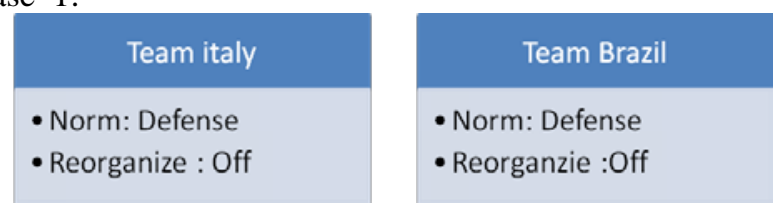

Figure 4.

In the Case 1, we allow the simulation run for a time period of 11400 clock cycles.

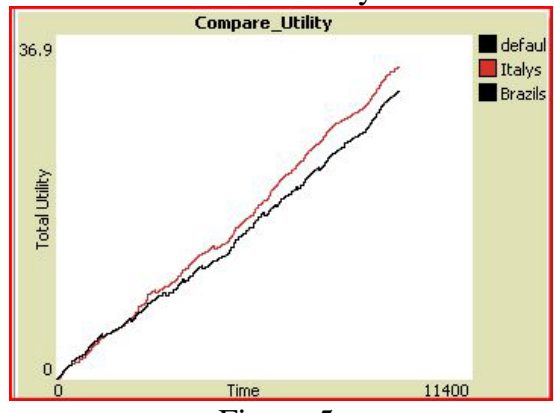

Figure 5.

The resultant graph in figure 5 compares utilities between the respective teams. Both teams norms were set to "Defense".

Case 2:

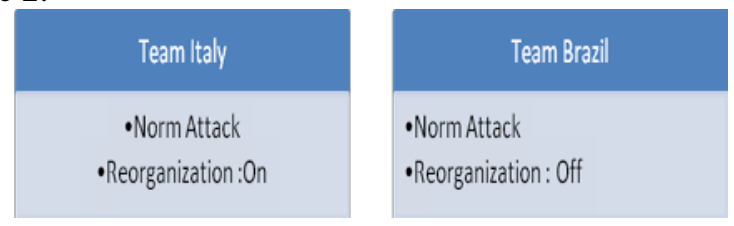

Figure 6.

In this case we set the norm for both teams in "attack" mode. Reorganization switch is turned on for Italy and it is turned off for Brazil. There are three players in Defense and MidField department and there are four players playing on the 
forward department. The resultant graph of utilities for teams at a time cycle of 11400 clock cycles is shown in Figure 7.

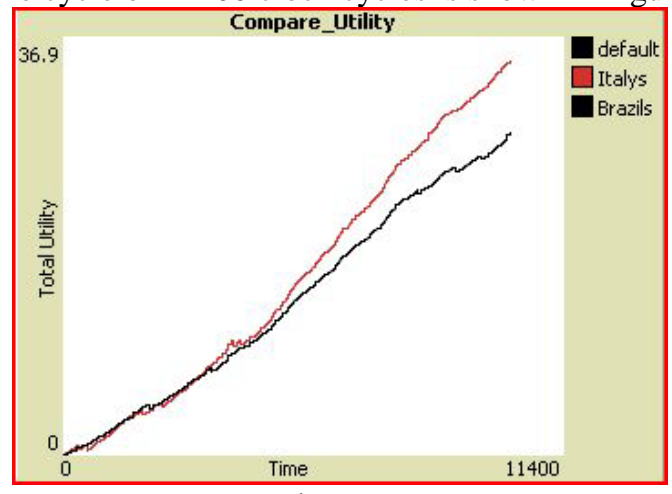

Figure 7.

At the time when the readings are noted the graph shows, Team Italy's utility 36.9 and Team Brazil's utility at 31.2. Due to the implementation of the reorganization algorithm the Team Italy has a considerable advantage over the opponents.

\section{Conclusions}

We have made novel strides in managing massive agent organizations that contributes to development of our man on the loop paradigm. Our methodology allows for Moe to prescribe normative patterns of behavior, which in turn guide the reorganization process. Individuals most fit to play their current roles stay while others are directed to surroundings that augment their synergy. We have demonstrated with the popular game of simulated soccer but the results are generic and transfer to other domains.

\section{REFERENCES}

[1]S. Abdullah and V,. Lesser, "Multiagent reinforcement Learning and Self-Organization in Network Agent” In Proceedings of the 6th international joint conference on Autonomous agents and multiagent systems. 2007, pp. 172-179.

[2]M. Aicardi, F. Davoli, and R. Minciardi. Decentralized optimal control of markov chains with a common past information set. IEEE Transactions on Automatic Control, AC-32:10281031, 1987.

[3]D.S. Alberts, and R. Hayes, "Understanding command and control”, Command and Control Research Program (CCRP), available online at: www.dodccrp.org., 2006.

[4]M. Avvenuti, P. Corsini, P. Masci, and A. Vecchio, "Opportunistic computing for wireless sensor networks”, Proc. IEEE mobile ad hoc networking conference, 2007.
[5] A. Barabási, and R. Albert, "Emergence of Scaling in Randiom Networks”, Science 286: pp. 509-512, 1999.

[6]K. Chang, "The Performance of Edge Organizations in a Collaborative Task”, MS thesis, The Naval Postgraduate School, Monterey, CA., 2005

[7]H. Hexmoor and S. Pasupuletti, "Institutional versus Interpersonal Influences on Role Adoption", In AAMAS workshop: Representation and approaches for time-critical resource/role/task allocation, J. Modi and T. Wagner (Eds), Melbourne, Australia, 2003.

[8] M. Gladwell, “The Tipping Point: How Little Things Can Make a Big Difference”, Back Bay Books, 2002.

[9]E. Matson and S. DeLoach, “Using Dynamic Capability Evaluation to Organize a Team of Cooperative, Autonomous Robots”, Proc. 2003 International Conference on Artificial Intelligence (IC-AI '03), 2003.

[10] A. Rahman, and H. Hexmoor, 2004. "Negotiation to improve Role Adoption in Organizations”, In Proc. International Conference on Artificial Intelligence (IC-AI), pp. 476-480, CSREA Press, 2004.

[11] R. Albert, and A. Barabasi, "Statistical Mechanics of Complex Networks. In Review of Modern Physics (p. 74), 2002.

[12] K. Barber, and C. Martin, “Dynamic Reorganization of decision making groups. Proceedings of 5th autonomous agents”, 2001.

[13]K. Carley, K., and L. Gasser, “Computational organizational theory . Multiagent systems : A modern approach to distributed artificial intelligence”, pp. 299-300, 1999.

[14]L. Chaimowicz, M. Campos, and R. Kumar, "Dynamic Role Assignment for Cooperative Robots”, In IEEE Conference on Robotics and Automation , 2002.

[15] V. Dignum, “A Model for organizational interactions based on Agents, founded in Logic”, SIKS Dissertion Series 2004-1. 2004: Utrecht University , 2004. 
[16] V. Dignum, V. Furtado, F. Dignum, and A. Melo, "Towards a Simulation Tool for Evaluating Dynamic Reorganization of Agent Societies”, 2005.

[17]M. Gatson, M., and M. desJardins, “Agent Organized Networks for Dynamic Team Formation”, In AAMAS, 2005.

[18]M. Gatson, J. Simmons, and M. desJardins, Adapting "Network Structure for Efficient Team Formation”, In AAMAS Workshop on Learning and Evolution in Agent Based Systems, 2004.

[19] N. Glasser, and P. Marignot, "The Reorganization of societies of autonomous agents”, In MAAMAW , pp. 98-111, 1997.

[20] H. Handley, and A. Levis, “A Model to evaluate the effect of organizational adaption”, In H. Handley, and A. Levis., Computational and Mathematical Organization Theory 7 (pp. 5-44) Kluwer Academic. 2001.

[21]H. Hexmoor, B. McLaughlan, G. Tuli, "Natural Human Role in Supervising Complex Control Systems", In Journal of Experimental and Theoretical Artificial Intelligence, Taylor and Francis, pp. 59-77. 2008.

[22] K. Chang, P. Lehner, A. Levis, A. Zaidi, and Z. Zhao, “On Causal Influence Logic”. Technical report,George Mason university Centre for Excellence, 1994.

[23] P. Kazakos, and A. Zaidi, "An Algorithm for activation Timed Influence Nets”, In IEEE IRI 2008, July 13-15, 2008.

[24]E. Matson, and S. DeLoach, “An Organizational Model for Designing Adaptive Multiagent Systems”, In AAAI Workshop: Agent Organization, 2004.

[25]B. McLaughlan, and H. Hexmoor, "Influencing Massive Multi-agent Systems via Viral Trait Spreading”, In Third IEEE International Conference on Self-Adaptive and Self-Organizing Systems, 2009.

[26]E. Matson, and S. DeLoach, "Using Dynamic Capability Evaluation to Organize a Team of Cooperative Autonomous Robots”, Proc. the 2003 International Conference on AI , 2003.
[27] C. Shen, C. Choung, and P. Will, "Simulating SelfOrganization for Multi-Robor Systems”, In IEEE/RSJ International COnference on Intelligent Robots and Systems. 2002.

[28] G. Valetto, G. Kaise, and G. Kc. “A moblie agent approach to process-based dynamic adaption of complex software systems", In 8th European Workshop on Software process technology, pp. 102116. 2001.

[29] P. Yolum. And M. Singh, "Emergent Personalized Communities in Refferal Networks", IJCAI workshop on Intelligent Techniques for Web Personalization , 2003.

[30]Zaidi, and P. Papatoni-Kazakas, "Modelling with Influence Networks Using Influence Constants: A New Approach”, Proc. 2007 IEEE/SMC International Conference on Systems, Man, and Cybernetics, Montreal, Canada, 2007.

[31]A. Zhong and S. DeLoach, "An Investigation of Reorganization Algorithms”, In International Conference on Artifiial Inteligence, 2006. 